\title{
ARTí́CULO
}

\section{Variación estacional del nivel del mar en el Archipiélago de San Andrés, Providencia y Santa Catalina, Mar Caribe}

Seasonal sea level variation in the San Andrés, Providencia and Santa Catalina Archipelago, Caribbean Sea

\author{
Rafael Ricardo Torres-Parra ${ }^{1 *}$, Diana Margarita Sánchez-Reyes ${ }^{1 * *}$ \\ y Maritza Y. Moreno-Calderón ${ }^{1 * * *}$
}

\begin{abstract}
'Escuela Naval de Cadetes ‘Almirante Padilla', Isla de Manzanillo, Cartagena de Indias, Bolívar, Colombia. *rrtorresp@ yahoo.com, **dianamargaritasanchezreyes@ hotmail.com,***mayimoca@gmail.com

Abstract.- The sea level seasonal cycle was assessed in the San Andrés, Providencia and Santa Catalina Archipelago in the Caribbean Sea using 22 years of absolute dynamic topography. Large spatial variability was found in the sea level seasonal spectrum in the 9 most important geographical features that constitute the archipelago, depending on their meridional position and as consequence of different forcings for this cycle present in the Colombian Basin. The sea level annual variation has a minimal range of $2.7 \mathrm{~cm}$ in Quitasueño and a maximum range of $9.5 \mathrm{~cm}$ in Roncador. The seasonal cycle is maximum in July-August and minimum in January-March toward the north of the archipelago; on the contrary, the seasonal cycle is maximum in February-March and minimum in August-September toward the south of the archipelago. The seasonal cycle explains between the 3 and $23 \%$ of the monthly sea level variation in the archipelago and it is not steady in time; changes up to $3.6 \mathrm{~cm}$ were found in the amplitude and $83^{\circ}$ in the phase lag when the annual cycle was assessed fractionating the time series in 5-year segments. The seasonal cycle contribution must be included in the archipelago's risk assessment associated to sea level extreme values.
\end{abstract}

Key words: Sea level, seasonal variations, Caribbean Sea, Colombian Basin, San Andrés, Providencia and Santa Catalina Archipelago, Panamá-Colombia Gyre

Resumen.- El ciclo estacional del nivel del mar fue analizado en el Archipiélago de San Andrés, Providencia y Santa Catalina en el Mar Caribe con base en 22 años de topografía dinámica absoluta. Se encontró gran variación espacial en el espectro estacional del nivel del mar en las 9 principales geoformas que componen el archipiélago dependiendo de su posición meridional y como consecuencia de diferentes forzantes de este ciclo presentes en la Cuenca Colombia. La variación anual del nivel del mar tiene un rango mínimo de $2,7 \mathrm{~cm}$ en Quitasueño y un rango máximo de $9,5 \mathrm{~cm}$ en Roncador. En el norte del archipiélago el ciclo estacional es máximo entre julio y agosto, y mínimo entre enero y marzo; por el contrario en el sur del archipiélago el ciclo estacional es máximo entre febrero y marzo y mínimo entre agosto y septiembre. El ciclo estacional explica entre el 3 y el $23 \%$ de la variación mensual del nivel del mar en el archipiélago y no es constante en el tiempo; se encontraron cambios de hasta $3,6 \mathrm{~cm}$ en amplitud y de $83^{\circ}$ de fase cuando el ciclo anual fue evaluado fraccionando la serie de tiempo en segmentos de 5 años. La contribución del ciclo estacional debe incluirse para la evaluación de riesgos asociada a valores extremos del nivel del mar en el archipiélago.

Palabras clave: Nivel del mar, variaciones estacionales, Mar Caribe, Cuenca Colombia, Archipiélago de San Andrés, Providencia y Santa Catalina, Giro Panamá- Colombia

\section{INTRODUCCIÓN}

El estudio de la variación del nivel del mares de gran importancia para las comunidades costeras, por cuanto sus actividades diarias son afectadas por estos cambios. Estas variaciones también afectan ecosistemas costeros (e.g., estuarios y lagunas costeras) y deben de tenerse en cuenta para la estimación de riesgos de origen marino sobre el litoral. Estas variaciones se dan en diferentes frecuencias forzados por causas astronómicas, procesos oceanográficos y atmosféricos; los cambios en periodos cerca de 12 y 24 horas, corresponden principalmente a la marea generada por forzamientos astronómicos. Otras variaciones ocurren en periodos intranuales, estacionales, interanuales y de largo plazo como la tendencia del aumento del nivel medio del mar relacionado con el calentamiento global (Torres 2014). 
En lugares como el Mar Caribe, la micromarea (Torres \& Tsimplis 2011) es del mismo orden de magnitud que las variaciones estacionales del nivel del mar (Torres \& Tsimplis 2012), las cuales incluyen un componente semianual y otro anual. Por esta razón es importante conocer las variaciones estacionales del nivel del mar en esta cuenca, ya que el solo pronóstico de la marea no es suficiente para comprender el comportamiento del nivel del mar durante el año y por ende, como afecta las poblaciones costeras y sus actividades socioeconómicas, así como el impacto en los ecosistemas costeros.

El primer estudio global de la variación estacional del nivel del mar lo realizó Pattullo et al. (1955) a partir de datos de estaciones mareográficas. Otros estudios globales se han realizado desde entonces con datos de mareógrafos (e.g., Tsimplis \& Woodworth 1994), datos de altimetría satelital (e.g., Stammer 1997) y con modelos numéricos con asimilación de datos (e.g., Vinogradov et al. 2008). Al ser el nivel del mar una variable con impactos locales en las poblaciones y ecosistemas costeros, varios estudios han evaluado las variaciones estacionales a escala regional y local en lugares como el sur de Europa (Marcos \& Tsimplis 2007), el Mar del Norte (Plag \& Tsimplis 1999), el sur del Mar de China (Amiruddin et al. 2015), costa de Tailandia (Saramul \& Ezer 2014), el Pacífico Noroeste (Feng et al. 2015), entre otros.

Las variaciones estacionales del nivel del mar en el Caribe fueron descritas por Torres \& Tsimplis (2012) a partir de 27 estaciones mareográficas con series de tiempo entre 5 y 102 años de longitud, así como 18 años de altimetría satelital. Ellos encontraron gran variación espacial y temporal en los ciclos estacionales del nivel del mar, la cual explica en la costa hasta el 78\% de la variación mensual del nivel del mar en la región. Los datos de altimetría mostraron un ciclo anual con amplitud promedio de $4,7 \mathrm{~cm}$ y máxima de $8 \mathrm{~cm}$, en tanto que el ciclo semianual en promedio mostró una amplitud de 1,8 cm y máxima de $5 \mathrm{~cm}$. Ambos ciclos mostraron ser estadísticamente insignificantes (a un nivel del 1\%) en algunas áreas del Caribe. Así mismo, los autores lograron identificar forzantes dominantes del nivel del mar en la región, como el giro Panamá-Colombia en el sur de la Cuenca Colombia y la surgencia en la costa norte de Sur América.

Desafortunadamente en el Mar Caribe hay pocas series de tiempo obtenidas a través de mareógrafos, lo cual no permite conocer las variaciones locales del nivel del mar en importantes poblaciones costeras o zonas sensibles en esta región a los cambios del nivel del mar. Una de estas zonas es el Archipiélago de San Andrés, Providencia y Santa Catalina (en adelante ASyP), ubicado en la cuenca Colombia del Mar Caribe (Fig. 1). Este archipiélago tiene islas densamente pobladas como San
Andrés y Providencia, en las cuales es necesario conocer la variación del nivel del mar a diversas frecuencias para disminuir el riesgo ante amenazas de origen marino; en el Caribe estas amenazas incluyen la variación del nivel medio del mar, la cual en promedio es de 1,7 $\pm 1,3 \mathrm{~mm} \mathrm{a}^{-1}$ (Torres \& Tsimplis 2013), como niveles extremos generados por huracanes y frentes fríos, con una contribución de la variación estacional, la cual fue descrita por Torres \& Tsimplis (2014). Este archipiélago cuenta con algunas geoformas como islas, atolones, bancos y cayos que por su baja altura son sensibles a variaciones extremas o seculares del nivel del mar, las cuales podrían afectar severamente sus ecosistemas e incluso a futuro llegar a sumergirlas permanentemente.

Si bien el estudio de Torres \& Tsimplis (2012) analizó las variaciones estacionales del nivel del mar en la cuenca del Mar Caribe, no se han descrito estas variaciones de manera particular en el ASyP. Por lo tanto, el objetivo del presente artículo fue estudiar el componente anual y semianual del espectro del nivel del mar en la cuenca Colombia y en las 9 principales geoformas (en adelante denominadas islas) que conforman el Archipiélago (Fig. 1b) a partir de 22 años de altimetría satelital. Así mismo se analizó la variación temporal del ciclo estacional del nivel del mar en estas islas. No se usaron datos de mareógrafos in situ por cuanto no se contó con una serie de tiempo disponible para ninguna de las islas. Este estudio ofrece información para la evaluación de riesgos de origen marino en el ASyP que permita la toma de decisiones para la gestión del riesgo, construcción de protecciones costeras y mejorar el conocimiento de los ciclos estacionales de los ecosistemas marinos de esta región. Así mismo, se procuró establecer si el archipiélago, como unidad geomorfológica, presenta una variación estacional del nivel del mar similar en todas sus islas, o si por el contrario, la variación es diferente en respuesta a diversos forzantes oceanográficos o atmosféricos sobre la geografía de la Cuenca Colombia.

\section{MATERIALES Y MÉTODOS}

\section{Área de ESTUdio}

El Caribe es un mar semi-cerrado limitado en el sur y el oeste por Sur y Centro América respectivamente; el norte limita con las Antillas Mayores y el este por las Antillas Menores (Fig. 1). La variación atmosférica estacional es modulada por la posición de la Zona de Convergencia Inter-Tropical (ZCIT) que domina los vientos y precipitación (Poveda et al. 2006). El campo de vientos de superficie es dominado por los vientos alisios, con un chorro intenso y permanente en dirección oeste denominado el chorro bajo del Caribe o CLLJ (Caribbean Low Level Jet) por sus siglas en inglés (Amador 2008), con centro hacia las 

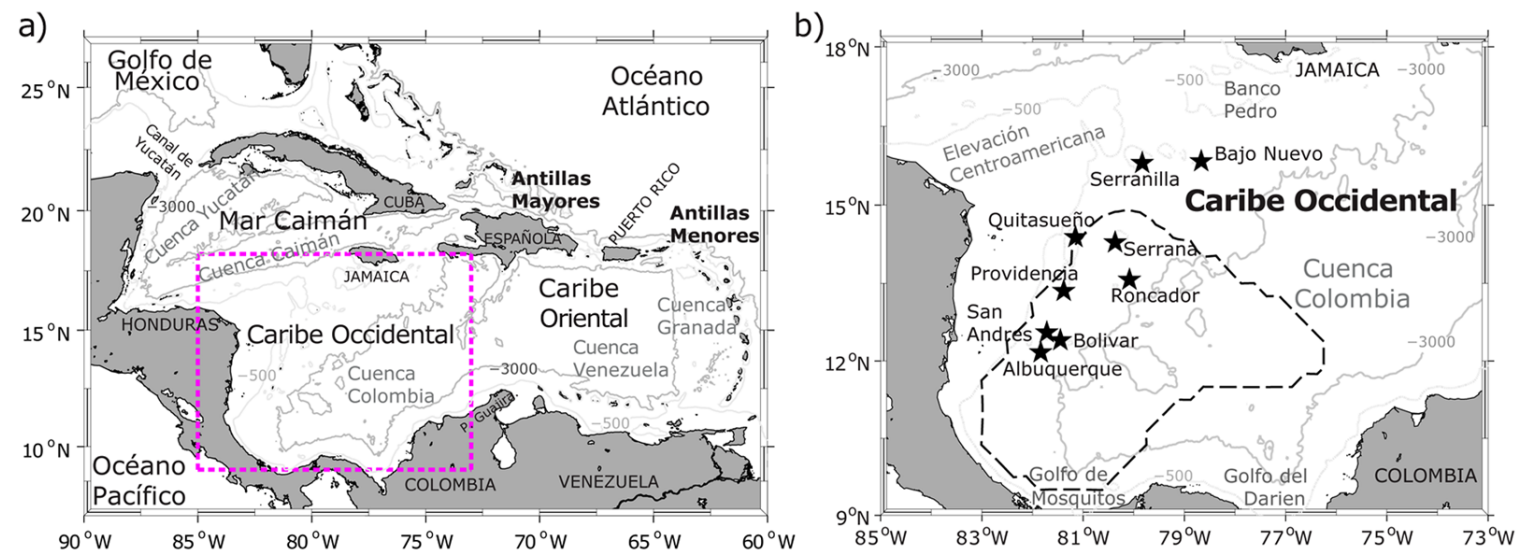

Figura 1. Área de estudio mostrando las isóbatas de $500 \mathrm{~m}$ y $3000 \mathrm{~m}$. a) el Mar Caribe. El recuadro punteado indica la Cuenca Colombia que se muestra en b) donde se señalan las 9 principales geoformas ( $\star$ ) del Archipiélago de San Andrés, Providencia y Santa Catalina. En línea punteada se muestra el límite del Giro Panamá-Colombia / The area of study with the $500 \mathrm{~m}$ and $3000 \mathrm{~m}$ isobaths. a) The Caribbean Sea; the dashed frame indicates the Colombian Basin which is shown in b) with the 9 most relevant geographical features $(\star)$ in the San Andrés, Providencia and Santa Catalina Archipelago. The dashed line indicates the Panamá-Colombia Gyre boundaries

latitudes entre $12^{\circ} \mathrm{N}$ y $15^{\circ} \mathrm{N}$. El CLLJ genera la surgencia en la costa norte de Sur América (Gordon 1967, Andrade \& Barton 2005) y es el principal forzante de los cambios estacionales del nivel del mar en esa sub-región del Caribe (Torres \& Tsimplis 2012).

La Corriente Caribe es la principal corriente en esta cuenca, la cual ingresa por las Antillas Menores con flujo en dirección este-oeste a lo largo de la costa norte de Sur América, hasta ingresar al Mar Caimán (Fig. 1) bordeando las costas de Centro América para salir hacia el norte por el Canal de Yucatán hacia el Golfo de México (Jouanno et al. 2008, Richardson 2005). El Giro Panamá-Colombia domina la circulación en el sur de la Cuenca Colombia (Andrade \& Barton 2000, Richardson 2005), modulando el nivel del mar en esta sub-región del Caribe (Torres \& Tsimplis 2012). La circulación del Mar Caribe es también afectada por remolinos o giros ciclónicos y anticiclónicos de mesoescala que recorren la cuenca (Chelton et al. 2007, Jouanno et al. 2008, Richardson 2005), los cuales contribuyen a niveles del mar extremos en la región (Torres \& Tsimplis 2014).

El ASyP se encuentra en el suroeste de la Cuenca Colombia. Esta cuenca limita por el este con la Cordillera Beata que se extiende de la isla Española hacia el suroeste y con la Elevación Centroamericana por el oeste (Fig. 1). Este archipiélago de origen volcánico está compuesto por las siguientes geoformas: 2 islas oceánicas San Andrés y Providencia (incluyendo su vecina Santa Catalina), las cuales están densamente pobladas; los atolones de Albuquerque, Bolívar (Courtown), Roncador,
Quitasueño y Serrana, así como los bancos coralinos de Bajo Nuevo y Serranilla (Díaz 2005). La posición de estas geoformas se presenta en la Tabla 2.

\section{Datos}

Se utilizaron mapas de topografía dinámica absoluta producidos por SSALTO/DUACS y distribuidos por AVISO. Se utilizaron los productos a escala global de post-proceso, con todos los satélites disponibles, empleando archivos con una resolución espacial en una grilla cartesiana de $0,25^{\circ}$. Estos mapas tienen correcciones de marea oceánica y terrestre, efecto de barómetro invertido, tropósfera seca y húmeda entre otros. La descripción más detallada del producto se puede encontrar en el manual del usuario SSALTO/DUACS (CLS-DOS-NT-06-034 del 2014/11/18). Se calcularon promedios mensuales en cada nodo de la grilla a partir de los datos diarios disponibles. La serie empleada cubre el periodo de enero de 1993 a diciembre del 2014.

Los datos de topografía dinámica absoluta son adecuados para la descripción de variaciones de nivel del mar costa afuera, en tanto que información de mareógrafos es útil para el análisis local. Comparaciones a nivel global del ciclo anual del nivel del mar en áreas costeras, calculado a partir de datos de altimetría y mareógrafos indican que las diferencias del valor cuadrático medio es menor o igual a $2 \mathrm{~cm}$ en el 76,4\% de los sitios (Ruiz Etcheverry et al. 2014). Al no contar con registros mareográficos disponibles para las islas del ASyP, el análisis local se realizó con información satelital teniendo en cuenta las 
Tabla 1. Resultados del modelo de regresión en las islas del Archipiélago de San Andrés, Providencia y Santa Catalina / Regression model results for the San Andrés, Providencia and Santa Catalina Archipelago islands ${ }^{\mathrm{a}}$

\begin{tabular}{|c|c|c|c|c|c|c|c|c|c|c|}
\hline \multirow[b]{2}{*}{$\begin{array}{l}\text { Nombre } \\
\text { Isla }\end{array}$} & \multirow{2}{*}{$\begin{array}{c}\text { Distancia } \\
\text { (Millas } \\
\text { Náticas) }\end{array}$} & \multirow{2}{*}{$\begin{array}{c}\text { Altura } \\
\text { Dinámica } \\
\text { Promedio }(\mathrm{cm})\end{array}$} & \multirow[b]{2}{*}{$\begin{array}{c}\text { Tendencia } \\
\mathrm{mm} \mathrm{a}^{-1}\end{array}$} & \multicolumn{2}{|c|}{ Ciclo Anual } & \multicolumn{2}{|c|}{ Ciclo Semi-anual } & \multirow[b]{2}{*}{$\begin{array}{l}\text { Rango } \\
(\mathrm{cm})\end{array}$} & \multirow{2}{*}{$\begin{array}{c}\text { Varianza } \\
\text { Explicada } \\
(\%)\end{array}$} & \multirow{2}{*}{$\begin{array}{c}\text { Desviación } \\
\text { Estándar } \\
(\mathrm{cm})\end{array}$} \\
\hline & & & & $\begin{array}{l}\text { Amplitud } \\
(\mathrm{cm})\end{array}$ & $\begin{array}{c}\text { Fase } \\
\left({ }^{\circ}\right)\end{array}$ & $\begin{array}{l}\text { Amplitud } \\
(\mathrm{cm})\end{array}$ & $\begin{array}{c}\text { Fase } \\
\left({ }^{\circ}\right)\end{array}$ & & & \\
\hline San Andrés & 7,0 & $-8,2 \pm 2,4$ & $4,1 \pm 1,0$ & $3,2 \pm 1,2$ & $60 \pm 21$ & & & 6,5 & 15,8 & 5,8 \\
\hline Albuquerque & 3,4 & $-10,8 \pm 2,3$ & $4,2 \pm 1,0$ & $3,4 \pm 1,2$ & $61 \pm 19$ & & & 6,8 & 20,1 & 5,6 \\
\hline Bolivar & 4,6 & $-10,0 \pm 2,7$ & $4,2 \pm 1,1$ & $4,5 \pm 1,4$ & $59 \pm 17$ & & & 9,0 & 23,2 & 6,7 \\
\hline Providencia & 1,6 & $-6,5 \pm 2,6$ & $3,7 \pm 1,1$ & $1,6 \pm 1,2$ & $66 \pm 53$ & & & 3,2 & 5,1 & 5,8 \\
\hline Roncador & 4,3 & $-4,2 \pm 3,7$ & $2,9 \pm 1,6$ & $2,9 \pm 1,8$ & $66 \pm 37$ & $2,6 \pm 1,5$ & $14 \pm 38$ & 9,5 & 10,5 & 8,5 \\
\hline Serrana & 5,5 & $-0,7 \pm 3,8$ & $2,7 \pm 1,6$ & & & $2,8 \pm 1,6$ & $16 \pm 37$ & 5,5 & 6,6 & 8,5 \\
\hline Quitasueño & 1,5 & $-3,9 \pm 2,6$ & $3,1 \pm 1,1$ & & & $1,3 \pm 1,3$ & $42 \pm 46$ & 2,7 & 3,3 & 5,7 \\
\hline Serranilla & 5,1 & $8,7 \pm 2,9$ & $2,5 \pm 1,2$ & $3,2 \pm 1,5$ & $215 \pm 26$ & $1,5 \pm 1,3$ & $29 \pm 46$ & 7,8 & 13,6 & 6,8 \\
\hline Bajo Nuevo & 3,5 & $14,6 \pm 4,6$ & $1,7 \pm 2,0$ & $2,8 \pm 2,3$ & $214 \pm 47$ & & & 5,7 & 5,0 & 10,4 \\
\hline
\end{tabular}

${ }^{a}$ Errores al $95 \%$ de nivel de confianza. Distancia entre las islas y el nodo del mapa de topografía dinámica más cercano. Solo se muestran valores de los armónicos estadísticamente significativos. Rango es la diferencia entre el mayor y menor valor de la suma de los armónicos anual y semi-anual. Desviación estándar de la serie original de topografía dinámica mensual

Tabla 2. Variación temporal de los armónicos estacionales ${ }^{\mathrm{a}}$ / Temporal variability of seasonal harmonics ${ }^{\mathrm{a}}$

\begin{tabular}{|c|c|c|c|c|c|c|c|c|c|c|}
\hline \multirow[b]{2}{*}{$\begin{array}{l}\text { Nombre } \\
\text { Isla }\end{array}$} & \multirow[b]{2}{*}{$\begin{array}{l}\text { Latitud } \\
\text { Norte }\end{array}$} & \multirow[b]{2}{*}{$\begin{array}{l}\text { Longitud } \\
\text { Oeste }\end{array}$} & \multicolumn{4}{|c|}{ Ciclo anual } & \multicolumn{4}{|c|}{ Ciclo Semi-anual } \\
\hline & & & $\begin{array}{c}\text { Segmentos } \\
\text { Significativos } \\
(\%)\end{array}$ & $\begin{array}{l}\text { Máxima } \\
\text { Amplitud } \\
(\mathrm{cm})\end{array}$ & $\begin{array}{c}\text { Rango } \\
\text { Amplitud } \\
\text { (cm) }\end{array}$ & $\begin{array}{l}\text { Rango } \\
\text { Fase } \\
\left({ }^{\circ}\right)\end{array}$ & $\begin{array}{c}\text { Segmentos } \\
\text { Significativos } \\
(\%)\end{array}$ & $\begin{array}{l}\text { Máxima } \\
\text { Amplitud } \\
(\mathrm{cm})\end{array}$ & $\begin{array}{c}\text { Rango } \\
\text { Amplitud } \\
\text { (cm) }\end{array}$ & $\begin{array}{l}\text { Rango } \\
\text { Fase } \\
\left({ }^{\circ}\right)\end{array}$ \\
\hline San Andrés & $12,55^{\circ}$ & $81,72^{\circ}$ & 77 & 4,9 & 2,3 & 53 & 0 & & & \\
\hline Albuquerque & $12,17^{\circ}$ & $81,84^{\circ}$ & 84 & 5,3 & 2,8 & 52 & 6 & 2,3 & 0,4 & 24 \\
\hline Bolívar & $12,40^{\circ}$ & $81,45^{\circ}$ & 93 & 6,5 & 3,6 & 45 & 0 & & & \\
\hline Providencia & $13,35^{\circ}$ & $81,38^{\circ}$ & 11 & 3,3 & 0,7 & 75 & 1 & 2,5 & 0,0 & 2 \\
\hline Roncador & $13,57^{\circ}$ & $80,08^{\circ}$ & 13 & 4,6 & 1,1 & 46 & 0 & & & \\
\hline Serrana & $14,28^{\circ}$ & $80,37^{\circ}$ & 0 & & & & 0 & & & \\
\hline Quitasueño & $14,38^{\circ}$ & $81,15^{\circ}$ & 0 & & & & 0 & & & \\
\hline Serranilla & $15,80^{\circ}$ & $79,83^{\circ}$ & 60 & 5,1 & 2,0 & 67 & 2 & 2,9 & 0,2 & 16 \\
\hline Bajo Nuevo & $15,83^{\circ}$ & $78,67^{\circ}$ & 14 & 5,2 & 1,1 & 83 & 0 & & & \\
\hline
\end{tabular}

siguientes condiciones encontradas por Torres \& Tsimplis (2012): (i) en las estaciones analizadas en el sur de la Cuenca Colombia el ciclo estacional no es afectado por el efecto del barómetro invertido; (ii) en el Mar Caribe el ciclo estacional calculado a partir de mediciones in situ no mostró diferencias al calculado a partir de datos satelitales; (iii) la variación temporal de los ciclos estacionales del nivel del mar observado en periodos de 5 años en el Caribe con datos de altimetría mostraron ser similares con aquellos derivados de mareógrafos sobre el mismo periodo de tiempo. No obstante, es importante a futuro contar con series de tiempo de nivel del mar en estas islas para corroborar los resultados descritos en este trabajo. Estos autores también encontraron que series de tiempo de 20 años son adecuadas para obtener una representación estable del ciclo estacional medio en el Caribe, lo cual se cumple con los 22 años de altimetría empleados en este artículo. La batimetría fue tomada de ETOPO2 con resolución de 2 min de grado.

\section{Metodología}

Los armónicos anuales y semi-anuales del nivel del mar se estimaron usando la siguiente regresión lineal ajustada a la serie de tiempo mensual

$Z_{0}(\mathrm{t})=\overline{Z_{0}}+a t+C_{1} \cos \left[\frac{2 \pi}{12} t-\varphi_{1}\right]+C_{2} \cos \left[\frac{2 \pi}{6} t-\varphi_{2}\right]$

donde el nivel del mar mensual observado $\left(Z_{0}\right)$ varía en el tiempo $(t)$ y es explicado por un valor medio de largo plazo $\left(\overline{Z_{0}}\right)$, una tendencia lineal $(a)$ y ciclos anual y semi-anual, los cuales son descritos por su amplitud $\left(C_{1}, C_{2}\right)$ y fase $\left(\varphi_{1}, \varphi_{2}\right)$ respectivamente. Las fases son medidas en grados con respecto al primero de enero $\left(0^{\circ}\right)$. Todos los errores se estimaron a un $95 \%$ de intervalo de confianza.

La significancia estadística de los ciclos anual y semi-anual fueron analizados a través de un análisis de la varianza 
(ANDEVA) usando la prueba F para determinar si cada término de la variación estacional mejoraba al $1 \%$ la varianza obtenida por el modelo. Esto se calculó a partir de la razón entre la variancia del residuo del modelo sobre la variancia de la serie original después de restarle el promedio y la tendencia a la serie de tiempo.

Los ciclos estacionales de las islas del ASyPfueron evaluados en el nodo de topografía dinámica absoluta más cercano a cada isla. Los cambios temporales del ciclo estacional fueron calculados a partir de la aplicación de la Ecuación (1) a segmentos de 5 años que avanzaban de a un mes hasta llegar al final de la serie de tiempo de 22 años. Los periodos utilizados fueron de 5 años al minimizar la gran variabilidad de los ciclos anuales y semi-anuales que se encuentra cuando son calculados a partir de segmentos anuales, dando amplitudes y fases más estables (Tsimplis \& Woodworth 1994).

\section{Resultados}

\section{Variación estacional Del NIVEL Del MAR EN LA CUENCA Colombia}

Los resultados de topografía dinámica en la Cuenca Colombia se presentan en la Figura 2. El cero en el campo promedio (Fig. 2a) indica el promedio espacial de la topografía dinámica en el Mar Caribe (58,8 cm). Teniendo en cuenta que la topografía dinámica mide el nivel del mar a partir del geoide, esta figura indica las corrientes permanentes en esta cuenca. Entre el Golfo de Mosquitos y Jamaica se observa un gradiente en el promedio de topografía dinámica de aproximadamente $40 \mathrm{~cm}$. Entre mayor sea el gradiente más intensa es la circulación. Al norte de la isolínea del promedio de la cuenca $(0 \mathrm{~cm})$ la topografía dinámica promedio aumenta cerca de 20 $\mathrm{cm}$ hasta el Banco Pedro (Fig. 1). Este gradiente es indicativo de la circulación intensa y permanente de la Corriente Caribe fluyendo desde la Cuenca Colombia hacia el Mar Caimán. Nótese la limitación que impone la topografía del fondo (Elevación Centroamericana y Bajo Pedro) a la Corriente Caribe. Al sur de la Cuenca Colombia están las menores alturas dinámicas promedio. El gradiente asociado a las 2 depresiones en altura dinámica son indicativo de la circulación ciclónica (inversa a las manecillas del reloj) permanente del Giro PanamáColombia.

La amplitud del ciclo anual no es significativa en el área al sur de Jamaica y al este de la Elevación Centroamericana y Banco Pedro (Fig. 2c). En contraste, la amplitud es mayor a 7 $\mathrm{cm}$ cerca de las costas continentales de Colombia al norte de $\operatorname{los} 11^{\circ} \mathrm{N}$, y mayor a $5 \mathrm{~cm}$ sobre la Elevación Centroamericana y en 2 sectores al sur de la Cuenca Colombia. La Figura 2d indica que el ciclo anual tiene su valor máximo entre septiembre y octubre $\left(240^{\circ}-300^{\circ}\right)$, salvo en inmediaciones de Jamaica donde el máximo es en agosto y en el sur de la Cuenca Colombia con valor máximo desde comienzos del año hasta marzo $\left(0^{\circ}-\right.$ $\left.90^{\circ}\right)$. El ciclo anual con máximas a comienzo del año corresponden a la ubicación del Giro Panamá-Colombia, cuyos límites (fases menores a $120^{\circ}$ ) se muestran en la Figura $1 \mathrm{~b}$.

El ciclo semi-anual es estadísticamente insignificante en aproximadamente una tercera parte de la Cuenca Colombia (Fig. 2e). Este ciclo tiene amplitud máxima de casi $5 \mathrm{~cm}$ entre latitudes $12^{\circ} \mathrm{N}$ y $13^{\circ} \mathrm{N}$ al este de la cuenca, el cual en la medida que se extiende hacia el oeste va disminuyendo su amplitud. La fase del ciclo semi-anual va retardándose de este a oeste de la cuenca (Fig. 2f). Inicia hacia los $74^{\circ} \mathrm{W}$ y en el Golfo del Darién con valores máximos a finales de abril/octubre $\left(-130^{\circ}\right)$; en el sur de la cuenca y al norte de los $12^{\circ} \mathrm{N}$ hasta $\sim 78^{\circ} \mathrm{W}$ los valores se retrasan alcanzando los máximos en mayo/noviembre $\left(-120^{\circ}\right.$ a $\left.-60^{\circ}\right)$. En el centro de la cuenca el ciclo se sigue retardando hacia el noroeste, teniendo sus máximos en junio/ diciembre $\left(-60^{\circ}\right.$ a $\left.0^{\circ}\right)$, luego en julio/enero $\left(0^{\circ}\right.$ a $\left.60^{\circ}\right)$ para finalmente tener sus máximos a comienzos de agosto/febrero $\left(\sim 75^{\circ}\right)$ en inmediaciones de Jamaica. Al oeste de la Elevación Centroamericana se observa un ciclo semi-anual estadísticamente significativo de hasta $2 \mathrm{~cm}$ de amplitud con máximos en febrero/agosto.

En la Cuenca Colombia, se tiene una diferencia espacial de $\sim 70 \%$ en la explicación que de la varianza mensual de nivel del mar dan los ciclos estacionales (Fig. 2b). Estos ciclos explican más del $70 \%$ de la variación mensual al oeste sobre las aguas someras de la Elevación Centroamericana y cerca de las costas continentales de Colombia al norte de los $11^{\circ} \mathrm{N}$, donde los ciclos anual y semi-anual tienen las mayores amplitudes. La variación estacional explica menos del 10\% de la varianza en 2 áreas. La primera al sur de Jamaica, donde los ciclos estacionales son estadísticamente insignificantes, posiblemente al ser enmascarados por variaciones en otras frecuencias, teniendo en cuenta que esta área presenta la mayor desviación estándar de la serie mensual de topografía dinámica (no mostrada), indicando a su vez la mayor variación del nivel del mar en la cuenca. La segunda área es en el borde de la Elevación Centroamericana hasta las costas de Centroamérica, en el límite oeste del Giro Panamá-Colombia, donde el ciclo anual tiene amplitudes menores a los $3 \mathrm{~cm}$ y el ciclo semi-anual no es significativo.

\section{VARIACIÓN ESTACIONAL DEL NIVEL DEL MAR EN LAS ISLAS DEL ASYP}

Por falta de datos de mareógrafos locales, se analiza la variación del nivel del mar en las 9 islas del ASyP con base en la información de altimetría. La máxima distancia entre el nodo 
a) Campo promedio topografía dinámica $(\mathrm{cm})$

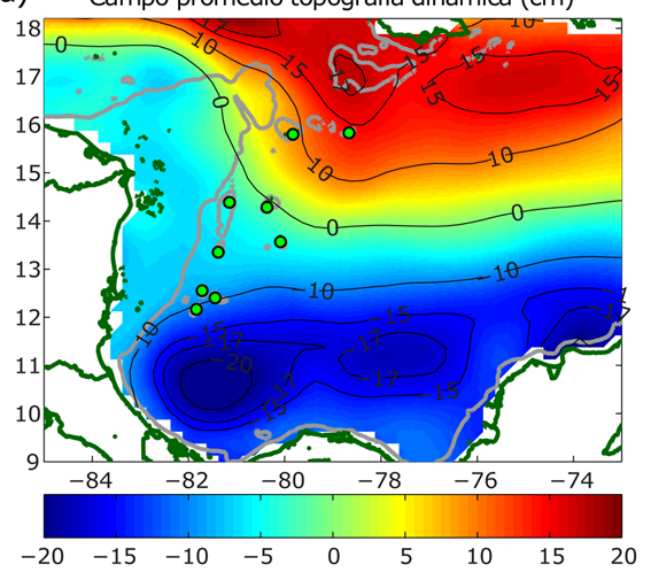

c)

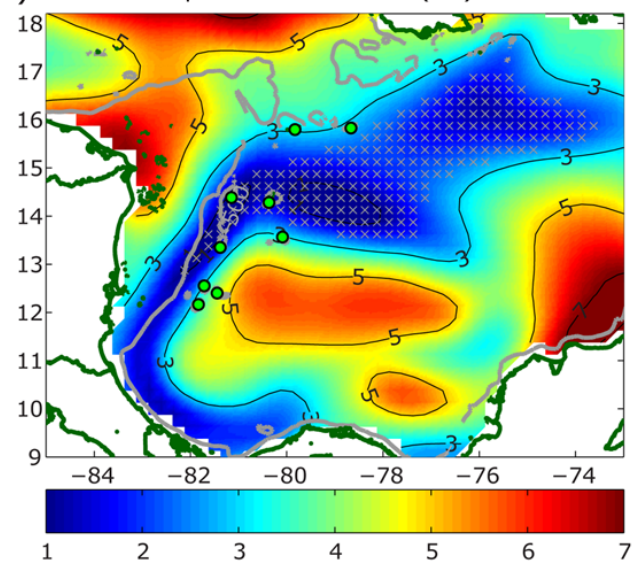

e) Amplitud del ciclo semianual $(\mathrm{cm})$

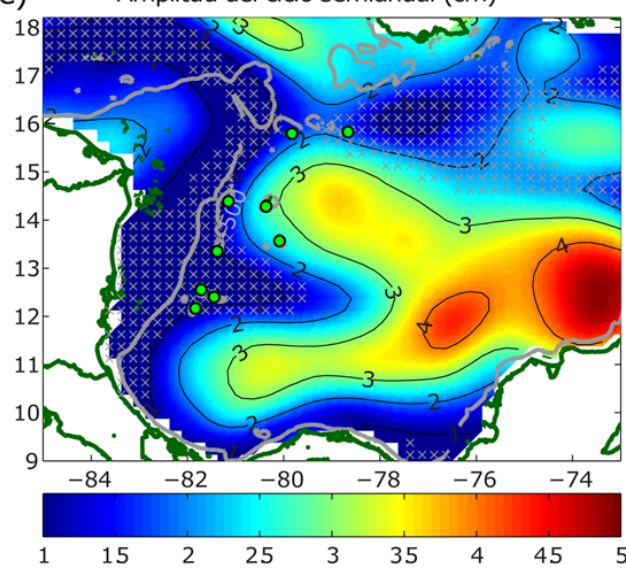

b) Explicación de la varianza ciclo estacional (\%)

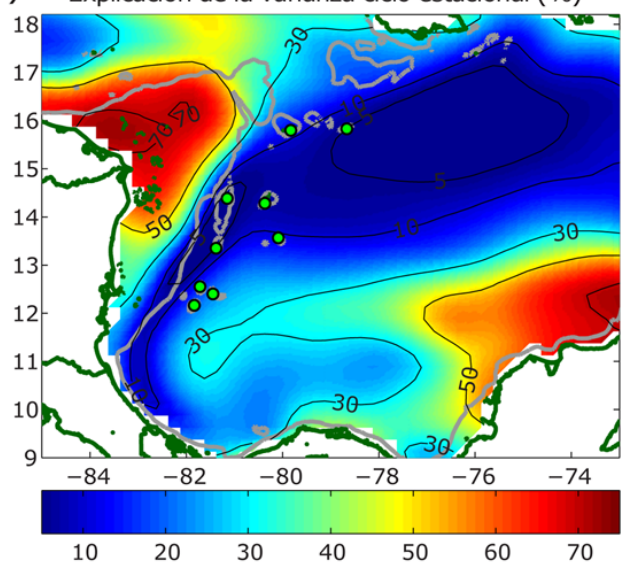

d)

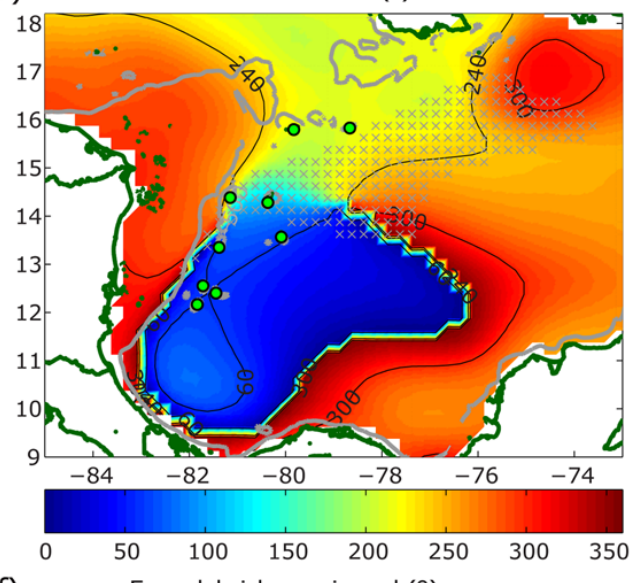

f)

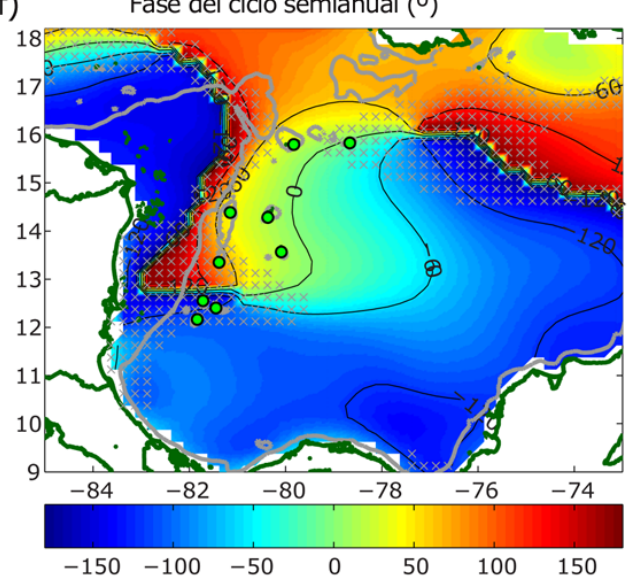

Figura 2. Resultados de altimetría en la Cuenca Colombia en el periodo 1993-2014. Círculos verdes corresponden a las 9 principales geoformas del Archipiélago de San Andrés, Providencia y Santa Catalina. La línea gris indica la isóbata de $\mathbf{5 0 0} \mathrm{m}$. En achurado gris los componentes armónicos estadísticamente insignificantes. a) Campo promedio de la topografía dinámica $(\mathrm{cm})$. b) Porcentaje de varianza mensual explicada por el ciclo estacional. c) Amplitud anual (cm). d) Fase anual (grados). e) Amplitud semi-anual (cm). f) Fase semi-anual (grados); a los valores mayoresa $180^{\circ}$ se les restó $360^{\circ}$ para mejorar la visualización en el centro de la cuenca / Altimetry results in the Colombian Basin for the period $1993-2014$. The 9 most relevant geographical features in the San Andrés, Providencia and Santa Catalina Archipelago are shown in green circles. $500 \mathrm{~m}$ isobath in gray line. Gray stippling where the harmonic components are statistically insignificant. a) Mean dynamic topography field (cm). b) Seasonal harmonics percent explained variance. c) Annual amplitude (cm). d) Annual phase (degrees). e) Semi-annual amplitude (cm). f) Semi-annual phase (degrees); $360^{\circ}$ were subtracted from values over $180^{\circ}$ to improve the visualization in the center of the basin 
más cercano de los mapas de topografía dinámica y cada una de las islas es de 7 millas náuticas (isla de San Andrés - Tabla 1). El máximo gradiente del valor promedio de topografía dinámica se da entre Bajo Nuevo $(14,6 \mathrm{~cm})$, la isla más al norte, y Albuquerque $(-10,8 \mathrm{~cm})$, la isla más al sur, en una distancia de 287,1 millas náuticas (Tabla 1).

Adicionalmente, en la Tabla 1 se presenta la tendencia en cada una de las islas a partir de los 22 años de datos de altimetría, teniendo en cuenta que esta es una de las variables del modelo de regresión (Ec. 1). La única tendencia no significativa es la de Bajo Nuevo. Todas las tendencias significativas están por encima de 1,7 $\pm 1,3 \mathrm{~mm} \mathrm{a}^{-1}$ valor promedio para el Mar Caribe con datos de altimetría en el periodo 1993-2010; no obstante por lo menos 40 años son requeridos para calcular tendencias estables en la región (Torres \& Tsimplis 2013). La mayor tendencia se observa en las 3 islas más al sur de la Cuenca Colombia, indicando que entre 1993 y 2014 el nivel del mar subió más de $9 \mathrm{~cm}$.

El ciclo anual no tiene un comportamiento homogéneo en el ASyP. El ciclo anual no es estadísticamente significativo en Serrana y Quitasueño, en tanto que es máximo en Bolívar con una amplitud 4,5 $\pm 1,4 \mathrm{~cm}$ (Tabla 1). Las 5 islas más al sur son afectadas por las variaciones estacionales del Giro PanamáColombia (Fig. 2d), con valores máximos entre finales de febrero y comienzos de marzo. Por el contrario, Serranilla y Bajo Nuevo al norte de la Cuenca Colombia, tiene un ciclo anual con máximos cerca de agosto. El ciclo semi-anual no es estadísticamente significativo en 5 de las 9 islas (Tabla 1); es significativo en las islas más al este, salvo por Bajo Nuevo (Fig. 2e). El máximo ciclo semi-anual se encuentra en Serrana con una amplitud de 2,8 $\pm 1,6 \mathrm{~cm}$. Todos los ciclos semi-anuales significativos muestran coherencia en la fase al tener máximos valores en enero y julio.

En la Figura 3 se presentan los ciclos estacionales de las 9 islas del archipiélago, solo teniendo en cuenta los componentes estadísticamente significativos. En las 5 islas en las cuales solo el ciclo anual es significativo se observa claramente el desfase de casi $180^{\circ}$ de Bajo Nuevo con respecto de las islas del sur del archipiélago. El ciclo semi-anual de Serrana tiene una amplitud similar a la del ciclo anual en Bajo Nuevo; el ciclo semi-anual de Quitasueño tiene una amplitud similar a la del ciclo anual en Providencia. Cuando los ciclos anual y semianual son estadísticamente significativos (Serranilla y Roncador), de la combinación de ellos resulta la variación real del nivel del mar mensual observada en estas islas. El mayor rango de los ciclos estacionales es de 9,5 cm y se encuentra en Roncador (Tabla 1), le sigue Bolívar donde solo el ciclo anual tiene un rango de $9 \mathrm{~cm}$, y en tercer lugar Serranilla con un rango en los ciclos estacionales de $7,8 \mathrm{~cm}$. Se puede observar la complejidad de la variación espacial de los ciclos estacionales del nivel del mar dependiendo de la ubicación meridional de las islas.
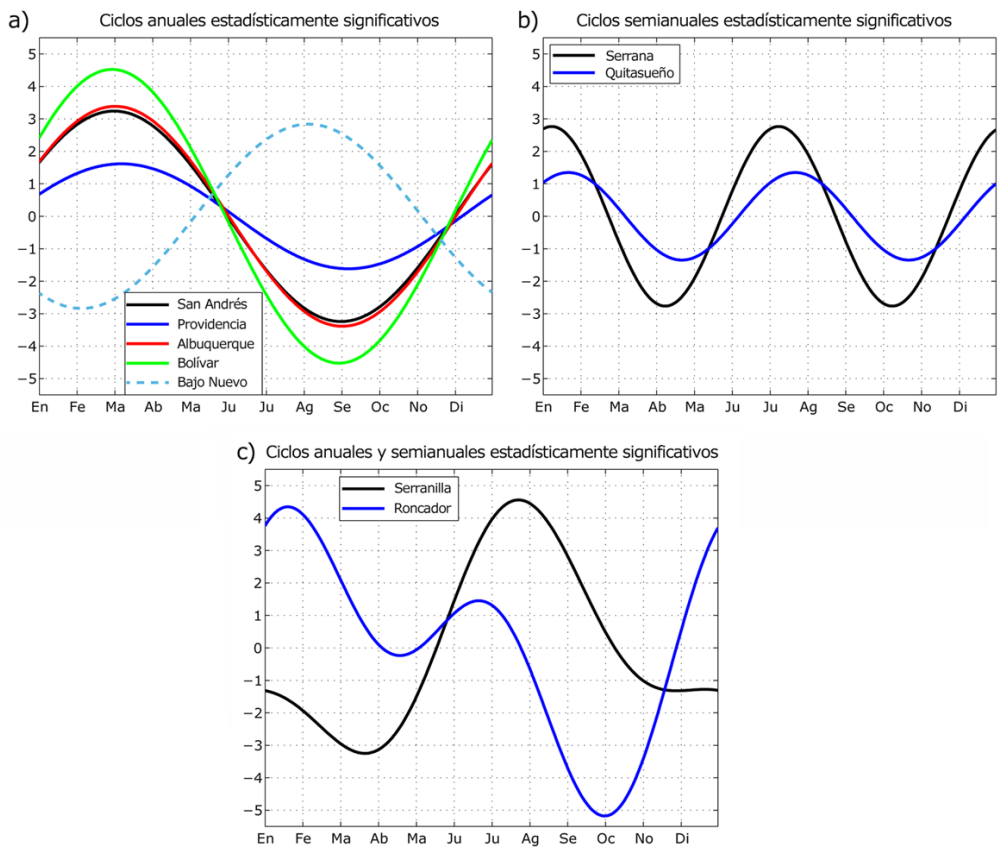

Figura 3. Armónicos estacionales estadísticamente significativos en las islas: a) ciclo anual, b) en ciclo semi-anual y c) los dos ciclos / Statistically significant seasonal harmonics in the islands: a) annual cycle, b) semi-annual cycle and c) both cycles 


\section{VARIACIÓN TEMPORAL DEL CICLO ESTACIONAL DEL NIVEL DEL MAR}

Los ciclos estacionales calculados con los 22 años de la serie de tiempo de altimetría son representativos de las variaciones dominantes en estas frecuencias, sin embargo, esto no significa que estos ciclos sean constantes en el tiempo. Los resultados de la variación en el tiempo del ciclo estacional evaluado en 204 segmentos de 5 años se presentan en la Tabla 2. El armónico anual no es constante en el tiempo; se confirma la insignificancia estadística de este ciclo en Serrana y Quitasueño, pues en ninguno de los segmentos quinquenales evaluados se encontró que el ciclo fuera estadísticamente significativo. En las otras 7 islas el porcentaje de ciclos anuales significativos varía del $93 \%$ de los segmentos en Bolívar (máxima amplitud del ciclo anual Tabla 1), al 11\% de los segmentos en Providencia (mínima amplitud del ciclo anual - Tabla1). En las 7 islas la máxima amplitud de los segmentos de 5 años (Tabla 2) fue mayor a la amplitud del ciclo anual evaluada con los 22 años de la serie completa (Tabla 1). El máximo rango del ciclo anual, que mide la diferencia entre la mayor y la menor amplitud de los segmentos estadísticamente significativos, es de 3,6 cm en Bolívar, y el menor rango es de 0,7 cm en Bolívar. La fase del armónico anual también presenta variaciones en los segmentos de 5 años con rangos entre los $45^{\circ}$ en Bolívar y $83^{\circ}$ en Bajo Nuevo. En la Figura 4 se observa como en las 3 islas más al sur, el armónico anual es significativo la mayor parte del tiempo a pesar de su alta variabilidad temporal. En estas 3 islas dada su cercanía, el armónico anual varía de manera similar, lo que no ocurre al compararse con Serranilla y Bajo Nuevo.

El ciclo semi-anual presenta variaciones temporales y diferencias con los ciclos calculados con 22 años de datos. En la Tabla 1 se muestran las 5 islas que no tienen ciclo semi-anual estadísticamente significativo evaluados en toda la serie de tiempo; de estas en Albuquerque y Providencia se encontró que el 6 y $1 \%$ de los segmentos de 5 años tienen armónicos semi-anuales significativos. Por el contrario, de las 4 islas que mostraron ciclo semi-anual estadísticamente significativo al evaluar la totalidad de la serie de tiempo, 3 de ellas (Roncador, Serrana y Quitasueño) no tuvieron un solo segmento de 5 años en el cual el armónico semi-anual fuera significativo; en el caso de Serranilla, solo el $2 \%$ de los segmentos fueron significativos. Esto indica que el armónico semi-anual del nivel del mar en el ASyP no tiene una energía dominante por lo cual presenta gran variabilidad temporal y espacial.

\section{DiscusIón}

Los ciclos estacionales del nivel del mar mostrados para la cuenca Colombia (Fig. 2) son coincidentes con estudios globales calculados a partir de datos de mareógrafos y de altimetría (Tsimplis \& Woodworth 1994, Ruiz Etcheverry et al. 2014); la amplitud de los ciclos en esta cuenca son similares a la encontrada en la mayoría de regiones. Los ciclos estacionales descritos para el ASyP en este artículo (Fig. 3), de acuerdo con el conocimiento de los autores se presentan por primera vez, por lo cual no pueden ser comparados con estudios previos; consecuentemente estos datos ofrecen nueva información para conocer la variabilidad del nivel del mar en esta frecuencia en estas islas y cayos, lo cual es útil para la toma de decisiones relacionadas con el manejo de la zona costera, entender variaciones hidrodinámicas anuales y su influencia en los ecosistemas, entre otras.

Se mostró que la circulación permanente en la Cuenca Colombia de acuerdo con los gradientes mostrados en la Figura 2a es dominada en el norte por la Corriente Caribe y en el sur por el Giro Panamá-Colombia, lo cual coincide con la descripción de la circulación del Mar Caribe presentada por Richardson (2005) a partir del análisis de boyas de deriva. Esto indica grandes diferencias en la circulación oceánica que afecta a las diferentes islas que componen el ASyP. El ciclo anual y semi-anual del nivel del mar en la Cuenca Colombia (Fig. 2) evaluados con las series de tiempo de topografía dinámica extendidas 4 años, confirman los resultados encontrados por Torres \& Tsimplis (2014) en el Mar Caribe.

El ASyP presenta una variación estacional del nivel del mar diferente en las islas que lo componen (Fig. 3) dependiendo de su posición geográfica. En todas las islas el ciclo estacional explica menos del $24 \%$ de la variación mensual del nivel del mar (Tabla 1). En las islas de Serranilla y Bajo Nuevo, al norte del archipiélago, los ciclos estacionales explican poco la varianza mensual ya sea por la energía en otras frecuencias intranuales que aportan remolinos al detenerse y desaparecer por las limitaciones topográficas (elevación Centroamericana y Bajo Pedro) encontradas en su tránsito de la Cuenca Colombia hacia el Mar Caimán, o por variaciones estacionales en la Corriente Caribe; del otro lado, en las islas hacia el centro y sur del archipiélago, la poca explicación de la varianza del ciclo estacional es consecuencia de la sobreposición del ciclo anual del Giro Panamá-Colombia forzado por el rotacional del estrés del viento y el ciclo estérico de la cuenca (Torres \& Tsimplis 2012).

En la Figura 3 se observa que el ciclo estacional en Serranilla y Bajo Nuevo al norte del archipiélago tiene nivel máximo entre julio y agosto, en tanto que el nivel es mínimo entre enero y marzo. La fase de este ciclo coincide con el ciclo de la subregión Caimán propuesta por Torres \& Tsimplis (2012), la cual es dominada por el intercambio estacional de 
flujos de densidad en superficie. Quitasueño y Serrana ubicadas en los $14^{\circ} \mathrm{N}$ de latitud, son las únicas islas con ciclo anual estadísticamente insignificante, posiblemente por estar en el límite el Giro Panamá Colombia (Fig. 2d) donde las variaciones estacionales del nivel del mar son menores (Fig. 2c), pero también por la influencia de la energía en otras frecuencias intranuales asociadas a los remolinos que transitan y desaparecen por la topografía del fondo en este sector (Torres \& Tsimplis 2012). En San Andrés, Providencia, Albuquerque y Bolívar la fase del ciclo anual (Fig. 3) es coincidente con la estacionalidad del giro Panamá-Colombia, indicando que las variaciones en el rotacional del estrés del viento (Torres \& Tsimplis 2012) dominan el ciclo estacional del nivel del mar en estas islas.

Se encontró gran variación temporal de los ciclos estacionales del nivel del mar en las islas del ASyP lo cual confirma lo descrito por Torres \& Tsimplis (2014) para el Mar Caribe. La coherencia en los cambios temporales del ciclo anual de San Andrés, Albuquerque y Bolívar, así como la disparidad con los cambios temporales en las islas al norte de Bajo Nuevo y Serranilla (Fig. 4) confirman la existencia de diferentes forzantes del ciclo anual del nivel del mar en este archipiélago.

a)

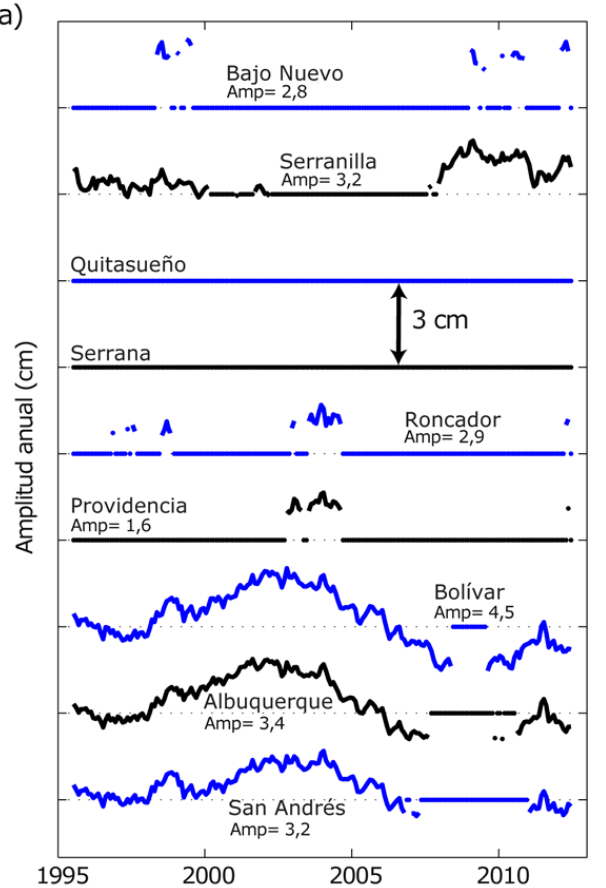

Este estudio describe la compleja variación estacional del nivel del mar en el ASyP a partir de 22 años de topografía dinámica. Es necesario a futuro verificar estos resultados mediante el análisis de series de tiempo in situ. Es igualmente importante evaluar como esta variación estacional del nivel del mar afecta los niveles extremos en las islas, para lo cual se debe complementar el estudio con el análisis de la micromarea y el efecto en el nivel del mar del tránsito de huracanes y frentes fríos, aportes de remolinos y el aumento secular del nivel del mar (Torres \& Tsimplis 2014). No obstante lo anterior, los resultados encontrados en este artículo indican los meses en que el ciclo del nivel del mar es máximo, época en que las islas serán más propensas a sufrir inundaciones en las zonas bajas como consecuencia de eventos extremos del nivel del mar. Este artículo indica además que en las islas más pobladas del archipiélago, el nivel medio del mar ha aumentado $9 \mathrm{~cm}$ en 22 años, lo cual se debe posiblemente a la disminución de la intensidad de rotación del giro Panamá-Colombia (Torres \& Tsimplis 2013). El conocimiento de todos estos componentes de la variación del nivel del mar en el Caribe es necesario para poder realizar una adecuada evaluación de esta amenaza en sitios de alta vulnerabilidad como el ASyP, lo cual es un primer paso para disminuir los riesgos en estas islas a causa de las variaciones del nivel del mar.

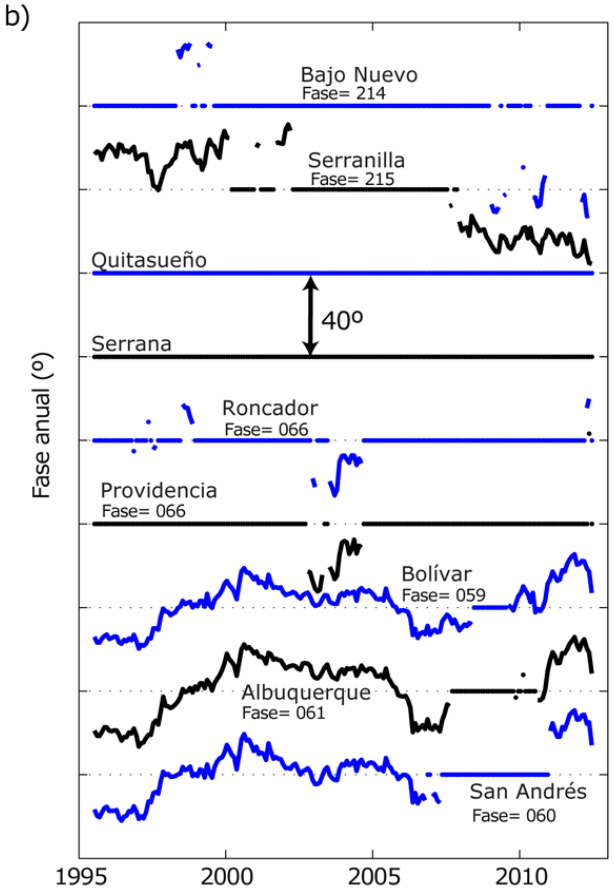

Figure 4. Variación temporal del armónico anual en las islas del Archipiélago de San Andrés, Providencia y Santa Catalina con respecto del valor obtenido de la serie de tiempo de 22 años (Tabla 1): a) Amplitud y b) Fase. Solo se muestran los valores de los segmentos de 5 años estadísticamente significativos / The temporal variability of the annual harmonic in the islands of the San Andrés, Providencia and Santa Catalina Archipelago referenced to the value obtained from the 22 year long time series (Table 1). a) Amplitude and b) Phase. Only the statistically significant 5 years segments are shown 


\section{Agradecimientos}

A los miembros de la facultad de oceanografía de la Escuela Naval de Cadetes 'Almirante Padilla' por su colaboración en la elaboración de este artículo, así como a los evaluadores anónimos por sus constructivas sugerencias.

\section{LITERATURA CITADA}

Amador J. 2008. The Intra-Americas Sea low-level jet. Annals of the New York Academy of Sciences 1146(1): 153-188. $<10.1196 /$ annals.1446.012>

Amiruddin A, I Haigh, M Tsimplis, F Calafat \& S Dangendorf. 2015. The seasonal cycle and variability of sea level in the South China Sea. Journal of Geophysical Research-Oceans 120(8): 5490-5513. <10.1002/2015jc 010923>

Andrade C \& E Barton. 2000. Eddy development and motion in the Caribbean Sea. Journal of Geophysical Research 105(C11): 26191-26201.<10.1029/2000jc000300>

Andrade C \& E Barton. 2005. The Guajira upwelling system. Continental Shelf Research 25(9): 1003-1022.

Chelton D, M Schlax, M Samelson \& R Szoeke. 2007. Global observations of large oceanic eddies. Geophysical Research Letters 34(15): L15606. <10.1029/2007gl030812>

Díaz M. 2005. Esquemas espaciales de zonación ecológica y morfología de las lagunas de los atolones y complejos arrecifales de un archipiélago oceánico del Caribe: San Andrés y Providencia (Colombia). Revista de la Academia Colombiana de Ciencias Exactas, Físicas y Naturales 29(112): 357-369.

Feng X, M Tsimplis, M Marcos, F Calafat, J Zheng, G Jordà \& P Cipollini. 2015. Spatial and temporal variations of the seasonal sea level cycle in the northwest Pacific. Journal of Geophysical Research-Oceans 120(10): 70917112. <10.1002/2015jc011154>

Gordon A. 1967. Circulation of the Caribbean Sea. Journal of Geophysical Research 72(24): 6207-6223. <10.1029/ JZ072i024p06207>

Jouanno J, J Sheinbaum, B Barnier, J Molines, L Debreu \& F Lemarié. 2008. The mesoscale variability in the Caribbean Sea. Part I: Simulations and characteristics with an embedded model. Ocean Modelling 23(3-4): 82-101.

Marcos M \& M Tsimplis. 2007. Forcing of coastal sea level rise patterns in the North Atlantic and the Mediterranean Sea. Geophysical Research Letters 34(18): L18604. $<10.1029 / 2007 \mathrm{gl} 030641>$

Pattullo J, W Munk, R Revelle \& E Strong. 1955. The seasonal oscillation in sea level. Journal of Marine Research 14(1): 88-155.
Plag H \& M Tsimplis. 1999. Temporal variability of the seasonal sea-level cycle in the North Sea and Baltic Sea in relation to climate variability. Global and Planetary Change 20(2-3): 173-203.

Poveda G, P Waylen \& R Pulwarty. 2006. Annual and interannual variability of the present climate in northern South America and southern Mesoamerica. Palaeogeography, Palaeoclimatology, Palaeoecology 234(1): 3-27.

Richardson P. 2005. Caribbean Current and eddies as observed by surface drifters. Deep Sea Research Part II: Topical Studies in Oceanography 52(3-4): 429-463.

Ruiz-Etcheverry LA, M Saraceno, AR Piola, G Valladeau \& OO Möller. 2015. A comparison of the annual cycle of sea level in coastal areas from gridded satellite altimetry and tide gauges. Continental Shelf Research 92: 87-97. <10.1016/ j.csr.2014.10.006>

Saramul S \& T Ezer. 2014. Spatial variations of sea level along the coast of Thailand: Impacts of extreme land subsidence, earthquakes and the seasonal monsoon. Global and Planetary Change 122: 70-81, <http://dx.doi.org/10.1016/j.gloplacha. 2014.08.012>

Stammer D. 1997. Steric and wind-induced changes in TOPEX/ POSEIDON large-scale sea surface topography observations. Journal of Geophysical Research 102(C9): 20987-21009. <10.1029/97jc01475>

Torres R. 2014. Sea-level change in the Caribbean Sea over the last century. Tesis Doctoral, Facultad de Ciencias Naturales y Ambientales, Universidad de Southampton, Southampton, $216 \mathrm{pp}$.

Torres R \& M Tsimplis. 2011. Tides and long-term modulations in the Caribbean Sea. Journal of Geophysical Research 116(C10):C10022, <10.1029/2011jc006973>

Torres R \& M Tsimplis. 2012. Seasonal sea level cycle in the Caribbean Sea. Journal of Geophysical Research, 117(C7): C07011. <10.1029/2012jc008159>

Torres R \& M Tsimplis. 2013. Sea-level trends and interannual variability in the Caribbean Sea. Journal of Geophysical Research-Oceans 118(6): 2934-2947.<10.1002/ jgrc.20229>

Torres R \& M Tsimplis. 2014. Sea level extremes in the Caribbean Sea. Journal of Geophysical Research-Oceans 119(8): 4714-4731. <10.1002/2014jc009929>

Tsimplis M \& P Woodworth. 1994. The global distribution of the seasonal sea level cycle calculated from coastal tide gauge data. Journal of Geophysical Research 99(C8): 1603116039. <10.1029/94jc01115>

Vinogradov S, R Ponte, P Heimbach \& C Wunsch. 2008. The mean seasonal cycle in sea level estimated from a dataconstrained general circulation model. Journal of Geophysical Research 113(C3): C03032. <10.1029/2007jc004496> 